\title{
Stable Perturbed Algorithms for a New Class of Generalized Nonlinear Implicit Quasi Variational Inclusions in Banach Spaces
}

\author{
Salahuddin, Mohammad Kalimuddin Ahmad \\ Department of Mathematics, Aligarh Muslim University, Aligarh, India \\ Email: salahuddin12@mailcity.com,ahmad_kalimuddin@yahoo.co.in
}

Received August 11, 2011; revised October 11, 2011; accepted October 20, 2011

\begin{abstract}
In this work, a new class of variational inclusion involving $T$-accretive operators in Banach spaces is introduced and studied. New iterative algorithms for stability for their class of variational inclusions and its convergence results are established.
\end{abstract}

Keywords: $T$-Accretive Operators; Variational Inclusions; Iterative Algorithms; Stability Conditions; Convergence; Strong Accretivity; Banach Spaces

\section{Introduction}

Variational inequality theory provides us with a simple, natural, general and unified framework for studying a wide range of unrelated problems arising in mechanics, physics, optimization and control theory nonlinear programming, economics, transportation, equilibrium and engineering sciences.

In recent years, variational inequality has been extended and generalized in different direction. A useful and important generalization of the variational inequality is called variational inclusions see [1-7].

Suppose $E$ is a real Banach space with dual space $E^{*}$, norm $\|$.$\| and dual pairing \langle.,\rangle,. 2^{E}$ is the family of all nonempty subsets of $E, C B(E)$ is the family of all nonempty closed bounded subset of $E$ and the generalized duality mapping $J_{q}: E \rightarrow 2^{E^{*}}$ is defined by

$$
\begin{gathered}
J_{q}(u)=\left\{f^{*} \in E^{*}:\left\langle u, f^{*}\right\rangle=\left\|f^{*}\right\| \cdot\|u\|,\right. \\
\left.\left\|f^{*}\right\|=\|u\|^{q-1}\right\}, \forall u \in E,
\end{gathered}
$$

where $q>1$ is a constant. In particular, $J_{2}$ is the usual normalized duality mapping. It is known that, in general $J_{q}(u)=\|u\|^{q-2} J_{2}(u)$ for all $u \neq 0$ and $J_{q}$ is simple single valued, if $E^{*}$ is strictly convex. The modulus of smoothness of $E$ is the function $\rho_{E}:[0, \infty) \rightarrow[0, \infty)$ defined by

$$
\rho_{E}(t)=\sup \left\{\frac{1}{2}(\|u+v\|+\|u-v\|)-1:\|u\| \leq 1,\|v\| \leq t\right\} .
$$

A Banach space $E$ is called uniformly smooth if $\lim _{t \rightarrow 0} \frac{\rho_{E}(t)}{t} \rightarrow 0 . E$ is called $q$-uniformly smooth, if there exists a constant $\rho>0$ such that

$$
\rho_{E}(t) \leq \rho t^{q}, q>1 .
$$

Note that $J_{q}$ is single valued, if $E$ is uniformly smooth. $\mathrm{Xu}$ and Roach [8] and $\mathrm{Xu}$ [9] proved the following results.

Lemma 1.1. Let $E$ be a real uniformly smooth Banach space. Then $E$ is q-uniformly smooth if and only if there exists a constant $c_{q}>0$ such that for all $u, v \in E$

$$
\|u+v\|^{q} \leq\|u\|^{q}+q\left\langle v, J_{q}(u)\right\rangle+c_{q}\|v\|^{q} .
$$

Definition 1.1. [10] Let $T: E \rightarrow E$ be a single-valued operator and $M: E \rightarrow 2^{E}$ be a multivalued operator. $M$ is said to be T-accretive if $M$ is accretive and $(T+\rho M)(E)=E$ hold for all $\rho>0$.

Remark 1.1. 1) From [11] it is easily establish that if $T=I$ (the identity map on $E$ ), then the definition of $I-$ accretive operator is that of m-accretive operator.

2) Example 2.1 in [11] shows that an $m$-accretive operator need not be $T$-accretive for some $T$.

Let $T: E \rightarrow E, N: E \times E \rightarrow E$ be two single valued mappings. Let $M: E \times E \rightarrow 2^{E}$ be a set-valued mapping such that for each fixed $t \in E, M(., t): E \rightarrow 2^{E}$ be a $T$-accretive operator. For given $f, g, p: E \rightarrow E$ are mappings, consider the following problem of finding $u \in E$ such that

$$
0 \in f(u)-N(u, u)+M(p u, g u),
$$


which is called the generalized nonlinear implicit quasi variational inclusions.

\section{Special Cases:}

1) If $E$ is a Hilbert space, then problem (1) is equivalent to finding $u \in E$ such that

$$
\begin{aligned}
& p u \in \operatorname{Dom}(M(., g u)) \\
& 0 \in f(u)-N(u, u)+M(p u, g u),
\end{aligned}
$$

which is called the generalized nonlinear implicit quasi variational inclusions, considered by Ding [12] and Fang et al. [13].

2) If $M(u, t)=M(u)$ for all $u, t \in E$, then problem (2) is equivalent to finding $u \in E$ such that

$$
\begin{aligned}
& p u \in \operatorname{Dom}(M) \\
& 0 \in f(u)-N(u, u)+M(p u),
\end{aligned}
$$

where $M: E \rightarrow 2^{E}$ is a maximal monotone mapping. The problem (3) was considered by Huang [14].

3) If $M(u, t)=\partial \varphi(u, t)$ for each $t \in E$, then problem (2) is equivalent to finding $u \in E$ such that

$$
\begin{aligned}
& p u \in \operatorname{Dom}(\partial \varphi(., g u)) \\
& \langle f(u)-N(u, u), v-p u\rangle \geq \varphi(p u, g u)-\varphi(v, g u),
\end{aligned}
$$

where $\varphi: E \times E \rightarrow R \cup\{+\infty\}$ such that for each $t \in E$, $\varphi(., t): E \rightarrow R \cup\{+\infty\}$ is a proper convex lower semicontinuous function with

$$
\operatorname{Range}(p) \cap \operatorname{Dom}(\partial \varphi(., t)) \neq \varnothing .
$$

The problem (4) was considered by Ding [15] for $g$ to be an identity mapping.

4) If $f=0$ and $g$ is the identity mapping, then problem (1) is equivalent to finding $u \in E$ such that

$$
\begin{aligned}
& p u \in \operatorname{Dom}(M(., u)) \\
& 0 \in-N(u, u)+M(p u, u),
\end{aligned}
$$

which is called the generalized strongly nonlinear implicit quasi variational inclusions, considered by Shim et al. [16].

Remark 1.2. For a suitable choice of $f, g, p, N, M$ and the space E, a number of classes of variational inequalities, complementarity problems and the variational inclusions can be obtained as special cases of the generalized nonlinear implicit quasivariational inclusions (1).

Let $T: E \rightarrow E$ be a strictly monotone operator and $M: E \times E \rightarrow 2^{E}$ be a $T$-accretive operator. Fang and Huang [11] defined the resolvent operator

$$
J_{T, \rho}^{M(., t)}(v)=(T+\rho M(., t))^{-1}(v), \forall v \in E .
$$

By Theorem 2.2 in [11], we know that if $T: E \rightarrow E$ is a strictly accretive operator and $M: E \times E \rightarrow 2^{E}$ is a $T$-accretive operator, then the operator $J_{T, \rho}^{M(., t)}: E \rightarrow E$ is a single valued. From the proof of Theorem 2.3 in [11], it is easy to obtain the following result.

Lemma 1.2. [10] Let $T: E \rightarrow E$ be a strictly accretive operator with constant $\lambda>0$ and for each fixed $t \in E, M: E \times E \rightarrow 2^{E}$ be a T-accretive operator then the operator $J_{T, \rho}^{M(, t)}: E \rightarrow E$ is Lipschitz continuous with constant $\frac{1}{\lambda}$, i.e.,

$$
\left\|J_{T, \rho}^{M(., t)}(u)-J_{T, \rho}^{M(., t)}(v)\right\| \leq \frac{1}{\lambda}\|u-v\| \quad \forall u, v \in E .
$$

Lemma 1.3. Let $a$ and $b$ be two nonnegative real numbers. Then

$$
(a+b)^{q} \leq 2^{q}\left(a^{q}+b^{q}\right) .
$$

Proof.

$$
\begin{aligned}
(a+b)^{q} & \leq(2 \max \{a, b\})^{q}=2^{q}(\max \{a, b\})^{q} \\
& \leq 2^{q}\left(a^{q}+b^{q}\right) .
\end{aligned}
$$

Definition 1.2. Let $\left\{M^{n}\right\}$ and $M$ be a maximal monotone mappings for $n=0,1,2, \cdots$. The sequence $\left\{M^{n}\right\}$ is said to be graph converges to $M$ (write $M^{n} \stackrel{{ }_{G}}{\longrightarrow} M$ ) if for every $(u, v) \in \operatorname{Graph}(M)$, there exists a sequences $\left(u_{n}, v_{n}\right) \in \operatorname{Graph}\left(M^{n}\right)$ such that $u_{n} \rightarrow u$ and $v_{n} \rightarrow v$ as $n \rightarrow \infty$.

Lemma 1.4. [3] Let $M^{n}$ and $M$ be the maximal monotone mappings for $n=0,1,2, \cdots$. Then $M^{n} \stackrel{G}{\longrightarrow} M$ if and only if

$$
J_{\rho}^{M^{n}}(u) \rightarrow J_{\rho}^{M}(u),
$$

for every $u \in E$ and $\rho>0$, where $J_{\rho}^{M}=(I+\rho M)^{-1}$.

Lemma 1.5. Let $\left\{a_{n}\right\},\left\{b_{n}\right\}$ and $\left\{c_{n}\right\}$ be three sequences of nonnegative numbers satisfying the following condition. There exists a positive integers $n_{0}$ such that

$$
a_{n+1} \leq\left(1-t_{n}\right) a_{n}+b_{n} t_{n}+c_{n} \text {, for } n \geq n_{0}
$$

where $t_{n} \in[0,1], \sum_{n=0}^{\infty} t_{n}=+\infty, \lim _{n \rightarrow \infty} b_{n}=0$ and $\sum_{n=0}^{\infty} c_{n}<+\infty$. Then $a_{n} \rightarrow 0$ as $n \rightarrow \infty$.

Proof. Let $\sigma=\inf \left\{a_{n}: n \geq n_{0}\right\}$. Then $\sigma \geq 0$. Suppose that $\sigma>0$. Then $a_{n} \geq \sigma>0$ for all $n \geq n_{0}$. It follows from (11), that

$$
\begin{aligned}
a_{n+1} & \leq a_{n}-\sigma t_{n}+t_{n} b_{n}+c_{n} \\
& =a_{n}-\left(\frac{1}{2} \sigma-b_{n}\right) t_{n}-\frac{1}{2} \sigma t_{n}+c_{n}
\end{aligned}
$$

for all $n \geq n_{0}$. Since $b_{n} \rightarrow 0$ as $n \rightarrow \infty$, there exists $n_{1} \geq n_{0}$ such that

$$
\frac{1}{2} \sigma \geq b_{n}, \text { for all } n \geq n_{1} .
$$


Combining (11) and (12), we have

$$
a_{n+1} \leq a_{n}-\frac{1}{2} \sigma t_{n}+c_{n}
$$

for all $n \geq n_{1}$, which implies that

$$
\frac{1}{2} \sigma \sum_{n=n_{1}}^{\infty} t_{n} \leq a_{n_{1}}+\sum_{n=n_{1}}^{\infty} c_{n}<+\infty .
$$

This is a contradiction. Therefore, $\sigma=0$ and so there exists a subsequence $\left\{a_{n_{j}}\right\} \subset\left\{a_{n}\right\}$ such that $a_{n_{j}} \rightarrow 0$ as $j \rightarrow \infty$. It follows from (11) that

$$
a_{n_{j+1}} \leq a_{n_{j}}+b_{n_{j}} t_{n_{j}}+c_{n_{j}}
$$

and so $a_{n_{j+1}} \rightarrow 0$ as $j \rightarrow \infty$. A simple induction leads to $a_{n_{j+k}} \stackrel{a_{j+1}}{\rightarrow}$ as $j \rightarrow \infty$ for all $k \geq 1$ and this means that $a_{n} \rightarrow 0$ as $n \rightarrow \infty$. This completes the proof.

Lemma 1.6. Let $T: E \rightarrow E$ be a strictly accretive operator and for a fixed $t \in E, M: E \times E \rightarrow 2^{E}$ be a $T$-accretive operator in the first variable. If $u$ is a solution of the problem (1) if and only if

$$
g(u)=J_{T, \rho}^{M(., g u)}[T(p u)-\rho f(u)+\rho N(u, u)]
$$

where $\rho>0$ is a constant and

$$
J_{T, \rho}^{M(., g u)}=(T+\rho M(., g u))^{-1} .
$$

Proof. $u \in E$ is a solution of (1)

$$
\begin{aligned}
\Leftrightarrow & 0 \in f(u)-N(u, u)+M(p u, g u) \\
\Leftrightarrow & 0 \in \rho f(u)-\rho N(u, u)+\rho M(p u, g u) \\
\Leftrightarrow 0 \in & -T(p u)+\rho f(u)-\rho N(u, u)+T(p u) \\
& \quad+\rho M(p u, g u) \\
\Leftrightarrow & 0 \in-[T(p u)-\rho f(u)+\rho N(u, u)] \\
& \quad+(T+\rho M(., g u))(p u) \\
\Leftrightarrow & p u \equiv J_{T, \rho}^{M(., g u)}[T(p u)-\rho f(u)+\rho N(u, u)] .
\end{aligned}
$$

\section{Existence and Uniqueness Theorems}

In this section, we show the existence and uniqueness of solutions for the problem (1) in terms of Lemma 1.6.

Definition 2.1. Let $E$ be a real uniformly smooth $\mathrm{Ba}$ nach space and $T, g: E \rightarrow E$ be two single valued operators; $T$ is said to be

1) Accretive if

$$
\left\langle T u-T v, J_{q}(u-v)\right\rangle \geq 0, \quad \forall u, v \in E
$$

or, equivalently

$$
\left\langle T u-T v, J_{2}(u-v)\right\rangle \geq 0, \quad \forall u, v \in E ;
$$

2) Strictly accretive if $T$ is accretive and

$$
\left\langle T u-T v, J_{q}(u-v)\right\rangle=0, \text { if } u=v
$$

3) Strongly accretive if there exists a constant $r>0$ such that

$$
\left\langle T u-T v, J_{q}(u-v)\right\rangle \geq r\|u-v\|^{q}, \quad \forall u, v \in E
$$

or, equivalently

$$
\left\langle T u-T v, J_{2}(u-v)\right\rangle \geq r\|u-v\|^{2}, \quad \forall u, v \in E ;
$$

4) Lipschitz continuous if there exists a constant $s>0$ such that

$$
\|T u-T v\| \leq s\|u-v\|, \quad \forall u, v \in E ;
$$

5) Strongly accretive with respect to $g$ if there exists a constant $\gamma>0$ such that

$$
\left\langle T u-T v, J_{q}(g u-g v)\right\rangle \geq \gamma\|g u-g v\|^{q}, \quad \forall u, v \in E
$$

or, equivalently

$$
\left\langle T u-T v, J_{2}(g u-g v)\right\rangle \geq \gamma\|g u-g v\|^{2}, \quad \forall u, v \in E .
$$

Definition 2.2. Let $N: E \times E \rightarrow E$ and $g: E \rightarrow E$ be the maps, then

1) $N(.,$.$) is said to be strongly accretive with respect$ to first argument if there exists a constant $\alpha>0$ such that

$$
\left\langle N(u, .)-N(v, .), J_{q}(u-v)\right\rangle \geq \alpha\|u-v\|^{q}, \quad \forall u, v \in E
$$

or, equivalently

$$
\left\langle N(u, .)-N(v, .), J_{2}(u-v)\right\rangle \geq \alpha\|u-v\|^{2}, \quad \forall u, v \in E ;
$$

2) $N$ is said to relaxed accretive with respect to $g$ if there exists a constant $\zeta>0$ such that

$$
\begin{gathered}
\left\langle N(u, .)-N(v, .), J_{q}(g u-g v)\right\rangle \geq-\zeta\|g u-g v\|^{q}, \\
\forall u, v \in E ;
\end{gathered}
$$

3) $N$ is Lipschitz continuous in first argument if there exists a constant $\eta>0$ such that

$$
\|N(u, .)-N(v, .)\| \leq \eta\|u-v\|, \quad \forall u, v \in E .
$$

Theorem 2.1. Let $E$ be a q-uniformly smooth Banach space and $T: E \rightarrow E$ be a strongly accretive and Lipschitz continuous with positive constants $\gamma$ and $\delta$ respectively. Let $p: E \rightarrow E$ be the strongly accretive and Lipschitz continuous with positive constants $\alpha$ and $\beta$ respectively. Let $f, g: E \rightarrow E$ be Lipschitz continuous with positive constants $\mu$ and $\sigma$ respectively. Let $N: E \times E \rightarrow E$ be relaxed accretive with respect to $p_{1}$ in the first and second arguments with constants $\zeta>0$ and $\omega>0$ respectively, where $p_{1}: E \rightarrow E$ is defined by $p_{1}(u)=(T \circ p)(u)=T(p u)$ for all $u \in E$. Assume that $N$ is Lipschitz continuous with respect to first and second argument with constants $\tau>0$ and 
$\xi>0$ respectively. Let $M: E \times E \rightarrow 2^{E}$ be a T-accretive operator with second argument. If there exist constants $\rho>0$ and $\eta>0$ such that for all $u, v, w, \in E$

$$
\left\|J_{T, \rho}^{M(., g u)}(w)-J_{T, \rho}^{M(., g v)}(w)\right\| \leq \eta\|g u-g v\|
$$

and

$$
Q+\frac{1}{\lambda} P<1,
$$

where

$$
Q=\left(1-q \alpha+C_{q} \beta^{q}\right)^{1 / q}+\eta \sigma<1
$$

and

$$
\begin{aligned}
P= & \left(\delta^{q} \beta^{q}-q \rho(\zeta+\omega) \gamma^{q} \alpha^{q}+2^{q} C_{q} \rho^{q}\left(\tau^{q}+\xi^{q}\right)\right)^{1 / q} \\
& +\rho \mu<1 .
\end{aligned}
$$

Then the problem (1) has a unique solution $u^{*} \in E$.

Proof. By Lemma 1.6, it is enough to show that the mapping $F: E \rightarrow E$ has a unique fixed point $u^{*} \in E$ where $F$ is defined as follows.

$$
F(u)=u-p u+J_{T, \rho}^{M(., g u)}[T(p u)-\rho f(u)+\rho N(u, u)]
$$

for all $u \in E$. From (13) and (15), we have

$$
\begin{aligned}
\|F(u)-F(v)\|= & \left\|u-p u+J_{T, \rho}^{M(., g u)}[T(p u)-\rho f(u)+\rho N(u, u)]-\left\{v-p v+J_{T, \rho}^{M(., g v)}[T(p v)-\rho f(v)+\rho N(v, v)]\right\}\right\| \\
\leq & \|u-v-(p u-p v)\|+\left\|J_{T, \rho}^{M(., g u)}[T(p u)-\rho f(u)+\rho N(u, u)]-J_{T, \rho}^{M(., g u)}[T(p v)-\rho f(v)+\rho N(v, v)]\right\| \\
& +\left\|J_{T, \rho}^{M(., g u)}[T(p v)-\rho f(v)+\rho N(v, v)]-J_{T, \rho}^{M(., g v)}[T(p v)-\rho f(v)+\rho N(v, v)]\right\| \\
\leq & \|u-v-(p u-p v)\|+\frac{1}{\lambda}\|T(p u)-T(p v)+\rho N(u, u)-\rho N(v, v)\|+\frac{1}{\lambda}\|\rho f(u)-\rho f(v)\|+\eta\|g u-g v\| \\
\leq & \|u-v-(p u-p v)\|+\frac{1}{\lambda}\|T(p u)-T(p v)+\rho(N(u, u)-N(v, v))\|+\frac{\rho}{\lambda}\|f(u)-f(v)\|+\eta \sigma\|u-v\| .
\end{aligned}
$$

Now since $p$ is strongly accretive and Lipschitz continuous, we have

$$
\begin{aligned}
\|u-v-(p u-p v)\|^{q} & \leq\|u-v\|^{q}-q\left\langle p u-p v, J_{q}(u-v)\right\rangle+C_{q}\|p u-p v\|^{q} \\
& \leq\|u-v\|^{q}-q \alpha\|u-v\|^{q}+C_{q} \beta^{q}\|u-v\|^{q} \\
& \leq\left(1-q \alpha+C_{q} \beta^{q}\right)\|u-v\|^{q} \\
\Rightarrow \| u-v-(p u-p v) & \leq \sqrt[q]{1-q \alpha+C_{q} \beta^{q}}\|u-v\| .
\end{aligned}
$$

By the strong accretivity of $p$ with constant $\alpha$, we have

$$
\begin{aligned}
& \|p u-p v\|\|u-v\|^{q-1}=\|p u-p v\|\left\|J_{q}(u-v)\right\| \geq\left\langle p u-p v, J_{q}(u-v)\right\rangle \geq \alpha\|u-v\|^{q} \\
& \Rightarrow\|u-v\| \leq \frac{1}{\alpha}\|p u-p v\|
\end{aligned}
$$

that is

$$
\|p u-p v\| \geq \alpha\|u-v\| .
$$

Similarly, by the strong accretivity of $T$ with constant $\gamma$ we have

$$
\|T(p u)-T(p v)\| \geq \gamma\|p u-p v\| .
$$

By $\tau$-Lipschitz continuity of $N$ with respect to first argument and $\xi$-Lipschitz continuity of $N$ with respect to second arguments, we have

$$
\begin{aligned}
& \|N(u, u)-N(v, u)\| \leq \tau\|u-v\| . \\
& \|N(v, u)-N(v, v)\| \leq \xi\|u-v\| .
\end{aligned}
$$

Since $f$ is Lipschitz continuous, we get

$$
\|f(u)-f(v)\| \leq \mu\|u-v\| .
$$

Since $N: E \times E \rightarrow E$ is a relaxed accretive with respect to $p$ in the first and second argument with constant $\zeta>0$ and $\omega>0$ respectively, from (18) and (19), we have 


$$
\begin{aligned}
& \left\langle N(u, u)-N(v, u), J_{q}(T(p u)-T(p v))\right\rangle \\
& \geq-\zeta\|T(p u)-T(p v)\|^{q} \\
& \geq-\zeta \gamma^{q}\|p u-p v\|^{q} \\
& \geq-\zeta \gamma^{q} \alpha^{q}\|u-v\|^{q}
\end{aligned}
$$

and similarly

$$
\begin{aligned}
& \left\langle N(v, u)-N(v, v), J_{q}(T(p u)-T(p v))\right\rangle \\
& \geq-\omega \gamma^{q} \alpha^{q}\|u-v\|^{q} .
\end{aligned}
$$

From (23) and (24), Lipschitz continuity of $T, p$, Lemma 1.1 and Lemma 1.3, we have

$$
\begin{aligned}
& \|T(p u)-T(p v)+\rho(N(u, u)-N(v, v))\|^{q} \\
& =\|T(p u)-T(p v)\|^{q}+q \rho\left\langle N(u, u)-N(v, v), J_{q}(T(p u)-T(p v))\right\rangle+\rho^{q} C_{q}\|N(u, u)-N(v, v)\|^{q} \\
& \leq\|T(p u)-T(p v)\|^{q}-q \rho \zeta \gamma^{q} \alpha^{q}\|u-v\|^{q}-q \rho \omega \gamma^{q} \alpha^{q}\|u-v\|^{q}+2^{q} \rho^{q} C_{q}\left(\tau^{q}+\xi^{q}\right)\|u-v\|^{q} \\
& \leq\left[\beta^{q} \delta^{q}-q \rho(\zeta+\omega) \gamma^{q} \alpha^{q}+2^{q} \rho^{q} C_{q}\left(\tau^{q}+\xi^{q}\right)\right]\|u-v\|^{q} .
\end{aligned}
$$

Now from (16), (17), (22) and (25), we have

$$
\begin{aligned}
\|F(u)-F(v)\| & \leq\left[\left(1-q \alpha+C_{q} \beta^{q}\right)^{1 / q}+\frac{1}{\lambda}\left(\delta^{q} \beta^{q}-q \rho(\zeta+\omega) \gamma^{q} \alpha^{q}+2^{q} C_{q} \rho^{q}\left(\tau^{q}+\xi^{q}\right)\right)^{1 / q}+\frac{\rho \mu}{\lambda}+\eta \sigma\right]\|u-v\| \\
& \leq\left[Q+\frac{1}{\lambda} P\right]\|u-v\| \leq \theta\|u-v\|,
\end{aligned}
$$

where $Q=\left(1-q \alpha+C_{q} \beta^{q}\right)^{1 / q}+\eta \sigma$ and

$$
P=\left(\delta^{q} \beta^{q}-q \rho(\zeta+\omega) \gamma^{q} \alpha^{q}+2^{q} C_{q} \rho^{q}\left(\tau^{q}+\xi^{q}\right)\right)^{1 / q}+\rho \mu
$$

and $\theta=Q+\frac{1}{\lambda} P$.

From (14), we know that $0<\theta<1$. Therefore, there exists a unique $u^{*} \in E$ such that $F\left(u^{*}\right)=u^{*}$. This completes the proof.

\section{Perturbed Algorithms and Stability}

In this section, we construct some new perturbed iterative algorithms with errors for solving the problem (1) and prove the convergence and stability of the iterative sequences generated by the perturbed algorithms with errors.

Definition 3.1. Let $T$ be a self mapping of $E$ and $x_{n+1}=f\left(T, x_{n}\right)$ define an iterative procedure which yields a sequence of point $\left\{x_{n}\right\}$ in E. Suppose that $\{x \in E: T x=x\} \neq \varnothing$ and $\left\{x_{n}\right\}$ converges to a fixed point $x^{*}$ of T. Let $\left\{y_{n}\right\} \subset E$ and let

$$
\varepsilon_{n}=\left\|y_{n+1}-f\left(T, y_{n}\right)\right\| .
$$

1) If $\lim _{n \rightarrow \infty} \varepsilon_{n}=0$ implies that $\lim _{n \rightarrow \infty} y_{n}=x^{*}$, then the iterative procedure $\left\{x_{n}\right\}$ defined by $x_{n+1}=f\left(T, x_{n}\right)$ is said to be $T$-stable or stable with respect to $T$.

2) If $\sum_{n=0}^{\infty} \varepsilon_{n}<+\infty$ implies that $\lim _{n \rightarrow \infty} y_{n}=x^{*}$, then the iterative procedure $\left\{x_{n}\right\}$ is said to be almost $T$-stable. Some stability results of iterative algorithms have been established by several authors [17-19]. As was shown by Harder and Hicks [20], the study on the stability is both of the theoretical and numerical interest.

Remark 3.1. An iterative procedure $\left\{x_{n}\right\}$ which is $T$ stable is almost T-stable and an iterative procedure $\left\{x_{n}\right\}$ which is almost T-stable need not be T-stable [21].

Algorithm 3.1. Let $f, p, g, T: E \rightarrow E$ and $N: E \times E \rightarrow E$ be the five single valued mappings. Let $\left\{M^{n}\right\}$ of $M$ be the set-valued mapping from $E \times E$ into the power set of $E$ such that for each $t \in E, M^{n}(., t)$ and $M(., t)$ are T-accretive mappings and $M^{n}(., t) \stackrel{G}{\longrightarrow} M(., t)$. For any given $u_{0} \in E$, the perturbed iterative sequence $\left\{u_{n}\right\}$ with errors is defined as follows: 


$$
\begin{aligned}
& u_{n+1}=\left(1-\alpha_{n}\right) u_{n}+\alpha_{n}\left[v_{n}-p v_{n}+J_{T, \rho}^{M^{n}\left(., g v_{n}\right)}\left\{T\left(p v_{n}\right)-\rho f\left(v_{n}\right)-\rho N\left(v_{n}, v_{n}\right)\right\}\right]+\alpha_{n} e_{n}+l_{n} \\
& v_{n}=\left(1-\beta_{n}\right) u_{n}+\beta_{n}\left[w_{n}-p w_{n}+J_{T, \rho}^{M^{n}\left(., g w_{n}\right)}\left\{T\left(p w_{n}\right)-\rho f\left(w_{n}\right)-\rho N\left(w_{n}, w_{n}\right)\right\}\right]+\beta_{n} r_{n} \\
& w_{n}=\left(1-\gamma_{n}\right) u_{n}+\gamma_{n}\left[u_{n}-p u_{n}+J_{T, \rho}^{M^{n}\left(., g u_{n}\right)}\left\{T\left(p u_{n}\right)-\rho f\left(u_{n}\right)-\rho N\left(u_{n}, u_{n}\right)\right\}\right]+\gamma_{n} s_{n}
\end{aligned}
$$

for $n=0,1,2, \cdots$, where $\left\{\alpha_{n}\right\},\left\{\beta_{n}\right\}$ and $\left\{\gamma_{n}\right\}$ are three sequences in $[0,1],\left\{e_{n}\right\},\left\{r_{n}\right\},\left\{s_{n}\right\}$ and $\left\{l_{n}\right\}$ are four sequences in $E$ satisfying the following conditions:

$$
\begin{gathered}
\lim _{n \rightarrow \infty}\left\|s_{n}\right\|=\lim _{n \rightarrow \infty}\left\|e_{n}\right\|=\lim _{n \rightarrow \infty}\left\|r_{n}\right\|=0 \\
\sum_{n=0}^{\infty} \alpha_{n}=+\infty, \sum_{n=0}^{\infty}\left\|l_{n}\right\|<+\infty .
\end{gathered}
$$

From Algorithm 3.1, we obtain the following algorithm for the problem (3).

Algorithm 3.2. Let $f, p, T: E \rightarrow E$ and $N: E \times E \rightarrow E$ be four single valued mappings. Let $\left\{M^{n}\right\}$ and $M$ be T-accretive mappings from $E$ into the power of $E$ such that $M^{n} \stackrel{G}{\longrightarrow} M$. For any given $u_{0} \in E$, define the perturbed iterative sequences $\left\{u_{n}\right\}$ with errors as follows:

$$
\begin{aligned}
& u_{n+1}=\left(1-\alpha_{n}\right) u_{n}+\alpha_{n}\left[v_{n}-p v_{n}+J_{T, \rho}^{M^{n}}\left\{T\left(p v_{n}\right)-\rho f\left(v_{n}\right)+\rho N\left(v_{n}, v_{n}\right)\right\}\right]+\alpha_{n} e_{n}+l_{n} \\
& v_{n}=\left(1-\beta_{n}\right) u_{n}+\beta_{n}\left[w_{n}-p w_{n}+J_{T, \rho}^{M^{n}}\left\{T\left(p w_{n}\right)-\rho f\left(w_{n}\right)+\rho N\left(w_{n}, w_{n}\right)\right\}\right]+\beta_{n} r_{n} \\
& w_{n}=\left(1-\gamma_{n}\right) u_{n}+\gamma_{n}\left[u_{n}-p u_{n}+J_{T, \rho}^{M^{n}}\left\{T\left(p u_{n}\right)-\rho f\left(u_{n}\right)-\rho N\left(u_{n}, u_{n}\right)\right\}\right]+\gamma_{n} s_{n}
\end{aligned}
$$

$n=0,1,2, \cdots$, then $\left\{\alpha_{n}\right\},\left\{\beta_{n}\right\},\left\{\gamma_{n}\right\},\left\{e_{n}\right\},\left\{r_{n}\right\}$, $\left\{s_{n}\right\}$ and $\left\{l_{n}\right\}$ are same as Algorithm 3.1.

Remark 3.2. For a suitable choice of $T, f, g, N, M^{n}$ and $M$, Algorithm 3.1 reduces to several known Algorithms [22-24] as special cases.

Theorem 3.1. Let $f, p, g, T$ and $N$ be the same as in Theorem 2.1. Suppose that $\left\{M^{n}\right\}$ and $M$ are set-valued mappings from $E \times E$ into the power set of $E$ such that for each $t \in E, M^{n}(., t)$ and $M(., t)$ are T-accretive mappings and $M^{n}(., t) \stackrel{G}{\longrightarrow} M(., t)$. Assume that there exists constants $\rho>0$ and $\eta>0$ such that for each $u, v, z \in E$ and $n \geq 0$

$$
\begin{gathered}
\left\|J_{T, \rho}^{M(., u)}(z)-J_{T, \rho}^{M(., v)}(z)\right\| \leq \eta\|u-v\| \\
\left\|J_{T, \rho}^{M^{n}(., u)}(z)-J_{T, \rho}^{M^{n}(., v)}(z)\right\| \leq \eta\|u-v\|
\end{gathered}
$$

and the condition (13) holds. Let $\left\{y_{n}\right\}$ be a sequence in $E$ and define a sequence $\left\{\varepsilon_{n}\right\}$ of real numbers as follows:

$$
\begin{aligned}
& \varepsilon_{n}=\left\|y_{n+1}-\left\{\left(1-\alpha_{n}\right) y_{n}+\left[x_{n}-p x_{n}+J_{T, \rho}^{M^{n}\left(., g x_{n}\right)}\left\{T\left(p x_{n}\right)-\rho f\left(x_{n}\right)+\rho N\left(x_{n}, x_{n}\right)\right\}\right]+e_{n} \alpha_{n}+l_{n}\right\}\right\| \\
& x_{n}=\left(1-\beta_{n}\right) y_{n}+\beta_{n}\left[z_{n}-p z_{n}+J_{T, \rho}^{M^{n}\left(., g z_{n}\right)}\left\{T\left(p z_{n}\right)-\rho f\left(z_{n}\right)+\rho N\left(z_{n}, z_{n}\right)\right\}\right]+\beta_{n} r_{n} \\
& z_{n}=\left(1-\gamma_{n}\right) y_{n}+\gamma_{n}\left[y_{n}-p y_{n}+J_{T, \rho}^{M^{n}\left(., g y_{n}\right)}\left\{T\left(p y_{n}\right)-\rho f\left(y_{n}\right)+\rho N\left(y_{n}, y_{n}\right)\right\}\right]+\gamma_{n} s_{n}
\end{aligned}
$$

where $\left\{\alpha_{n}\right\},\left\{\beta_{n}\right\},\left\{\gamma_{n}\right\},\left\{e_{n}\right\},\left\{r_{n}\right\},\left\{s_{n}\right\}$ and $\left\{l_{n}\right\}$ are same sequences defined in Algorithm 3.1. Then the following holds:

1) The sequence $\left\{u_{n}\right\}$ defined by Algorithm 3.1 converges strongly to the unique solution $u^{*}$ of the problem (1).

2) If $\varepsilon_{n}=\alpha_{n} \Delta_{n}+\chi_{n}$ with $\sum_{n=0}^{\infty} \chi_{n}<+\infty$ and $\lim _{n \rightarrow \infty} \Delta_{n}=0$, then $\lim _{n \rightarrow \infty} y_{n}=u^{*}$.

3) If $\lim _{n \rightarrow \infty} y_{n}=u^{*}$ implies that $\lim _{n \rightarrow \infty} \varepsilon_{n}=0$.

Proof. Let $u^{*} \in E$ be the unique solution of the problem (1). It is easy to see that the conclusion (1) follows from the conclusion (2). Now we prove that (2) is true. It follows from Lemma 1.6 that 


$$
u^{*}=\left(1-\alpha_{n}\right) u^{*}+\alpha_{n}\left[u^{*}-p u^{*}+J_{T, \rho}^{M\left(\cdot, g u^{*}\right)}\left\{T\left(p u^{*}\right)-\rho f\left(u^{*}\right)+\rho N\left(u^{*}, u^{*}\right)\right\}\right] .
$$

From (27), (31) and (32), we have

$$
\begin{aligned}
& \left\|y_{n+1}-u^{*}\right\|=\left\|y_{n+1}-\left\{\left(1-\alpha_{n}\right) u^{*}+\alpha_{n}\left[u^{*}-p u^{*}+J_{T, \rho}^{M\left(., u^{*}\right)}\left\{T\left(p u^{*}\right)-\rho f\left(u^{*}\right)+\rho N\left(u^{*}, u^{*}\right)\right\}\right]\right\}\right\| \\
& \leq\left\|y_{n+1}-\left\{\left(1-\alpha_{n}\right) y_{n}+\alpha_{n}\left[x_{n}-p x_{n}+J_{T, \rho}^{M^{n}\left(., g x_{n}\right)}\left\{T\left(p x_{n}\right)-\rho f\left(x_{n}\right)+\rho N\left(x_{n}, x_{n}\right)\right\}\right]+\alpha_{n} e_{n}+l_{n}\right\}\right\| \\
& +\|\left(1-\alpha_{n}\right) y_{n}+\alpha_{n}\left[x_{n}-p x_{n}+J_{T, \rho}^{M^{n}\left(., g x_{n}\right)}\left\{T\left(p x_{n}\right)-\rho f\left(x_{n}\right)+\rho N\left(x_{n}, x_{n}\right)\right\}\right] \\
& -\left\{\left(1-\alpha_{n}\right) u^{*}+\alpha_{n}\left[u^{*}-p u^{*}+J_{T, \rho}^{M\left(,, g u^{*}\right)}\left\{T\left(p u^{*}\right)-\rho f\left(u^{*}\right)+\rho N\left(u^{*}, u^{*}\right)\right\}\right]\right\}\left\|+\alpha_{n}\right\| e_{n}\|+\| l_{n} \| \\
& \leq \varepsilon_{n}+\left(1-\alpha_{n}\right)\left\|y_{n}-u^{*}\right\|+\alpha_{n}\left\|x_{n}-u^{*}-\left(p x_{n}-p u^{*}\right)\right\| \\
& +\alpha_{n}\left\|J_{T, \rho}^{M^{n}\left(., g x_{n}\right)}\left\{T\left(p x_{n}\right)-\rho f\left(x_{n}\right)+\rho N\left(x_{n}, x_{n}\right)\right\}-J_{T, \rho}^{M\left(., g u^{*}\right)}\left[T\left(p u^{*}\right)-\rho\left(u^{*}\right)+\rho N\left(u^{*}, u^{*}\right)\right]\right\|+\alpha_{n}\left\|e_{n}\right\|+\left\|l_{n}\right\| \\
& \leq \varepsilon_{n}+\left(1-\alpha_{n}\right)\left\|y_{n}-u^{*}\right\|+\alpha_{n}\left\|x_{n}-u^{*}-\left(p x_{n}-p u^{*}\right)\right\| \\
& +\alpha_{n}\left\|J_{T, \rho}^{M^{n}\left(., x_{n}\right)}\left[T\left(p x_{n}\right)-\rho f\left(x_{n}\right)+\rho N\left(x_{n}, x_{n}\right)\right]-J_{T, \rho}^{M^{n}\left(., g x_{n}\right)}\left[T\left(p u^{*}\right)-\rho f\left(u^{*}\right)+\rho N\left(u^{*}, u^{*}\right)\right]\right\| \\
& +\alpha_{n}\left\|J_{T, \rho}^{M^{n}\left(,, g x_{n}\right)}\left[T\left(p u^{*}\right)-\rho f\left(u^{*}\right)+\rho N\left(u^{*}, u^{*}\right)\right]-J_{T, \rho}^{M^{n}\left(., g u^{*}\right)}\left[T\left(p u^{*}\right)-\rho f\left(u^{*}\right)+\rho N\left(u^{*}, u^{*}\right)\right]\right\| \\
& +\alpha_{n}\left\|J_{T, \rho}^{M^{n}\left(\cdot, g u^{*}\right)}\left[T\left(p u^{*}\right)-\rho f\left(u^{*}\right)+\rho N\left(u^{*}, u^{*}\right)\right]-J_{T, \rho}^{M\left(\cdot, g u^{*}\right)}\left[T\left(p u^{*}\right)-\rho f\left(u^{*}\right)+\rho N\left(u^{*}, u^{*}\right)\right]\right\|+\alpha_{n}\left\|e_{n}\right\|+\left\|l_{n}\right\| \\
& \leq \varepsilon_{n}+\left(1-\alpha_{n}\right)\left\|y_{n}-u^{*}\right\|+\alpha_{n}\left\|x_{n}-u^{*}-\left(p x_{n}-p u^{*}\right)\right\| \\
& +\frac{\alpha_{n}}{\lambda}\left\|T\left(p x_{n}\right)-T\left(p u^{*}\right)-\rho\left(f\left(x_{n}\right)-f\left(u^{*}\right)\right)+\rho\left(N\left(x_{n}, x_{n}\right)-N\left(u^{*}, u^{*}\right)\right)\right\| \\
& +\alpha_{n} \eta\left\|g x_{n}-g u^{*}\right\|+\alpha_{n} G_{n}+\alpha_{n}\left\|e_{n}\right\|+\left\|l_{n}\right\| \\
& \leq \varepsilon_{n}+\left(1-\alpha_{n}\right)\left\|y_{n}-u^{*}\right\|+\alpha_{n}\left\|x_{n}-u^{*}-\left(p x_{n}-p u^{*}\right)\right\| \\
& +\frac{\alpha_{n}}{\lambda}\left\|T\left(p x_{n}\right)-T\left(p u^{*}\right)+\rho\left(N\left(x_{n}, x_{n}\right)-N\left(u^{*}, u^{*}\right)\right)\right\| \\
& +\frac{\alpha_{n}}{\lambda} \rho\left\|f\left(x_{n}\right)-f\left(u^{*}\right)\right\|+\alpha_{n} \eta \sigma\left\|x_{n}-u^{*}\right\|+\alpha_{n} G_{n}+\alpha_{n}\left\|e_{n}\right\|+\left\|l_{n}\right\| \\
& \leq\left(1-\alpha_{n}\right)\left\|y_{n}-u^{*}\right\|+\alpha_{n}\left\|x_{n}-u^{*}-\left(p x_{n}-p u^{*}\right)\right\|+\frac{\alpha_{n}}{\lambda}\left\|T\left(p x_{n}\right)-T\left(p u^{*}\right)+\rho\left(N\left(x_{n}, x_{n}\right)-N\left(u^{*}, u^{*}\right)\right)\right\| \\
& +\frac{\alpha_{n} \rho \mu}{\lambda}\left\|x_{n}-u^{*}\right\|+\alpha_{n} \eta \sigma\left\|x_{n}-u^{*}\right\|+\alpha_{n}\left(G_{n}+\left\|e_{n}\right\|+\Delta_{n}\right)+\left(\left\|l_{n}\right\|+\chi_{n}\right)
\end{aligned}
$$

where

$$
G_{n}=\left\|J_{T, \rho}^{M^{n}\left(., g u^{*}\right)}\left[T\left(p u^{*}\right)-\rho f\left(u^{*}\right)+\rho N\left(u^{*}, u^{*}\right)\right]-J_{T, \rho}^{M\left(., g u^{*}\right)}\left[T\left(p u^{*}\right)-\rho f\left(u^{*}\right)+\rho N\left(u^{*}, u^{*}\right)\right]\right\| \rightarrow 0 .
$$

It follows from (17) and (25), that 


$$
\begin{gathered}
\left\|x_{n}-u^{*}-\left(p x_{n}-p u^{*}\right)\right\| \leq \sqrt[q]{1-\alpha q+C_{q} \beta^{q}}\left\|x_{n}-u^{*}\right\|, \quad\left\|f\left(x_{n}\right)-f\left(u^{*}\right)\right\| \leq \mu\left\|x_{n}-u^{*}\right\|, \\
\left\|T\left(p x_{n}\right)-T\left(p u^{*}\right)+\rho\left(N\left(x_{n}, x_{n}\right)-N\left(u^{*}, u^{*}\right)\right)\right\| \leq\left(\delta^{q} \beta^{q}-q \rho(\zeta+\omega) \gamma^{q} \alpha^{q}+2^{q} \rho^{q} C_{q}\left(\tau^{q}+\xi^{q}\right)\right)^{1 / q}\left\|x_{n}-u^{*}\right\| .
\end{gathered}
$$

Substituting (35) into (33), we have

$$
\begin{aligned}
\left\|y_{n+1}-u^{*}\right\| \leq & \left(1-\alpha_{n}\right)\left\|y_{n}-u^{*}\right\|+\alpha_{n} \theta\left\|x_{n}-u^{*}\right\| \\
& +\alpha_{n}\left(G_{n}+\left\|e_{n}\right\|+\Delta_{n}\right)+\left(\left\|l_{n}\right\|+\chi_{n}\right),
\end{aligned}
$$

$$
\begin{aligned}
\left\|x_{n}-u^{*}\right\| \leq & \left(1-\beta_{n}\right)\left\|y_{n}-u^{*}\right\| \\
& +\beta_{n} \theta\left\|z_{n}-u^{*}\right\|+\beta_{n}\left(G_{n}+\left\|r_{n}\right\|\right)
\end{aligned}
$$

and again

where

$$
\begin{aligned}
& \theta=Q+\frac{1}{\lambda} P \text { and } Q=\left(1-q \alpha+C_{q} \beta^{q}\right)^{1 / q}+\eta \sigma, \\
& P=\left(\delta^{q} \beta^{q}-q \rho(\zeta+\omega) \gamma^{q} \alpha^{q}+2^{q} \rho^{q} C_{q}\left(\tau^{q}+\xi^{q}\right)\right)^{1 / q}+\rho \mu .
\end{aligned}
$$

From (14), we know that $0<\theta<1$. Similarly, we have

$$
\begin{aligned}
\left\|z_{n}-u^{*}\right\| \leq & \left(1-\gamma_{n}\right)\left\|y_{n}-u^{*}\right\| \\
& +\gamma_{n} \theta\left\|y_{n}-u^{*}\right\|+\gamma_{n} G_{n}+\gamma_{n}\left\|s_{n}\right\| \\
\leq & \left(1-\gamma_{n}(1-\theta)\right)\left\|y_{n}-u^{*}\right\|+\gamma_{n} G_{n}+\gamma_{n}\left\|s_{n}\right\| \\
\leq & \left\|y_{n}-u^{*}\right\|+\gamma_{n}\left(G_{n}+\left\|s_{n}\right\|\right)
\end{aligned}
$$

where $\left(1-\gamma_{n}(1-\theta)\right) \leq 1$.

From (38) and (39), we get

$$
\begin{aligned}
\left\|x_{n}-u^{*}\right\| & \leq\left(1-\beta_{n}\right)\left\|y_{n}-u^{*}\right\|+\beta_{n} \theta\left\|y_{n}-u^{*}\right\|+\beta_{n} \gamma_{n} \theta\left(G_{n}+\left\|s_{n}\right\|\right)+\beta_{n}\left(G_{n}+\left\|r_{n}\right\|\right) \\
& \leq\left(1-\beta_{n}(1-\theta)\right)\left\|y_{n}-u^{*}\right\|+\beta_{n} \gamma_{n} \theta\left(G_{n}+\left\|s_{n}\right\|\right)+\beta_{n}\left(G_{n}+\left\|r_{n}\right\|\right) \\
& \leq\left\|y_{n}-u^{*}\right\|+\beta_{n} \gamma_{n} \theta\left(G_{n}+\left\|s_{n}\right\|\right)+\beta_{n}\left(G_{n}+\left\|r_{n}\right\|\right),
\end{aligned}
$$

since $\left(1-\beta_{n}(1-\theta)\right) \leq 1$. From (36) and (40), we get

$$
\begin{aligned}
\left\|y_{n+1}-u^{*}\right\| & \leq\left(1-\alpha_{n}\right)\left\|y_{n}-u^{*}\right\|+\alpha_{n} \theta\left\|y_{n}-u^{*}\right\|+\alpha_{n} \beta_{n} \gamma_{n} \theta^{2}\left(G_{n}+\left\|s_{n}\right\|\right)+\alpha_{n} \beta_{n} \theta\left(G_{n}+\left\|r_{n}\right\|\right)+\alpha_{n}\left(G_{n}+\left\|e_{n}\right\|+\Delta_{n}\right)+\left(\left\|l_{n}\right\|+\chi_{n}\right) \\
& \leq\left(1-\alpha_{n}(1-\theta)\right)\left\|y_{n}-u^{*}\right\|+(1-\theta) \alpha_{n} \cdot \frac{1}{1-\theta}\left[\beta_{n} \gamma_{n} \theta^{2}\left(G_{n}+\left\|S_{n}\right\|\right)+\beta_{n} \theta\left(G_{n}+\left\|r_{n}\right\|\right)+\left(G_{n}+\left\|e_{n}\right\|+\Delta_{n}\right)\right]+\left(\left\|l_{n}\right\|+\chi_{n}\right) .
\end{aligned}
$$

Let

$$
\begin{aligned}
& a_{n}=\left\|y_{n}-u^{*}\right\|, \quad c_{n}=\left\|l_{n}\right\|+\chi_{n}, \quad t_{n}=\alpha_{n}(1-\theta) \\
& b_{n}=\frac{1}{1-\theta}\left[\beta_{n} \gamma_{n} \theta^{2}\left(G_{n}+\left\|s_{n}\right\|\right)+\beta_{n} \theta\left(G_{n}+\left\|r_{n}\right\|\right)\right. \\
& +\left(G_{n}+\left\|e_{n}\right\|+\left\|\Delta_{n}\right\|\right) .
\end{aligned}
$$

We can write (42) as follows:

$$
a_{n+1} \leq\left(1-t_{n}\right) a_{n}+b_{n} t_{n}+c_{n} .
$$

From the assumption, we know that $\left\{a_{n}\right\},\left\{b_{n}\right\},\left\{c_{n}\right\}$ and $\left\{t_{n}\right\}$ satisfy the conditions of Lemma 1.5. This implies that $a_{n} \rightarrow 0$ and so $y_{n}-u^{*}$.

Next, we prove the condition (3). Suppose that $\lim _{n \rightarrow \infty} y_{n}=u^{*}$. It follows (28), (38) and (39) that $z_{n} \rightarrow u^{*}$ and $x_{n} \rightarrow u^{*}$. From (31), we have

$$
\begin{aligned}
\varepsilon_{n} & =\left\|y_{n+1}-\left\{\left(1-\alpha_{n}\right) y_{n}+\alpha_{n}\left[x_{n}-p x_{n}+J_{T, \rho}^{M^{n}\left(., g x_{n}\right)}\left(T\left(p x_{n}\right)-\rho f\left(x_{n}\right)+\rho N\left(x_{n}, x_{n}\right)\right)\right]+\alpha_{n} e_{n}+l_{n}\right\}\right\| \\
& \leq\left\|y_{n+1}-u^{*}\right\|+\alpha_{n}\left\|e_{n}\right\|+\left\|l_{n}\right\|+\left\|\left(1-\alpha_{n}\right) y_{n}+\alpha_{n}\left[x_{n}-p x_{n}+J_{T, \rho}^{M^{n}\left(., g x_{n}\right)}\left(T\left(p x_{n}\right)-\rho f\left(x_{n}\right)+\rho N\left(x_{n}, x_{n}\right)\right)\right]-u^{*}\right\| .
\end{aligned}
$$

As in the proof of (36), we have

$$
\begin{aligned}
& \left\|\left(1-\alpha_{n}\right) y_{n}+\alpha_{n}\left[x_{n}-p x_{n}+J_{T, \rho}^{M^{n}\left(\cdot, g x_{n}\right)}\left(T\left(p x_{n}\right)-\rho f\left(x_{n}\right)+\rho N\left(x_{n}, x_{n}\right)\right)\right]-u^{*}\right\| \\
& \leq\left(1-\alpha_{n}\right)\left\|y_{n}-u^{*}\right\|+\alpha_{n} \theta\left\|x_{n}-u^{*}\right\|+\alpha_{n} G_{n} .
\end{aligned}
$$

It follows from (43) and (44) that

$$
\varepsilon_{n} \leq\left\|y_{n+1}-u^{*}\right\|+\alpha_{n}\left\|e_{n}\right\|+\left\|l_{n}\right\|+\left(1-\alpha_{n}\right)\left\|x_{n}-u^{*}\right\|+\alpha_{n} \theta\left\|x_{n}-u^{*}\right\|+\alpha_{n} G_{n} .
$$


This implies that $\lim _{n \rightarrow \infty} \varepsilon_{n}=0$. This completes the proof.

Theorem 3.2. Let $f, p, N$ and $T$ be the same as in Theorem 3.1. Let $\left\{M^{n}\right\}$ and $M$ be T-accretive mappings from $E$ into the power set of $E$ such that $M^{n} \stackrel{G}{\longrightarrow} M$. Assume that there exists constant $\rho>0$ such that (14) hold. Let $\left\{y_{n}\right\}$ be a sequence in $E$ and define $\left\{\varepsilon_{n}\right\}$ as follows:

$$
\begin{aligned}
& \varepsilon_{n}=\left\|y_{n+1}-\left\{\left(1-\alpha_{n}\right) y_{n}+\alpha_{n}\left[x_{n}-p x_{n}+J_{T, \rho}^{M^{n}}\left(T\left(p x_{n}\right)-\rho f\left(x_{n}\right)+\rho N\left(x_{n}, x_{n}\right)\right)\right]+e_{n} \alpha_{n}+l_{n}\right\}\right\|, \\
& x_{n}=\left(1-\beta_{n}\right) y_{n}+\beta_{n}\left[z_{n}-p z_{n}+J_{T, \rho}^{M^{n}}\left\{T\left(p z_{n}\right)-\rho f\left(z_{n}\right)+\rho N\left(z_{n}, z_{n}\right)\right\}\right]+\beta_{n} r_{n} \\
& z_{n}=\left(1-\gamma_{n}\right) y_{n}+\gamma_{n}\left[y_{n}-p y_{n}+J_{T, \rho}^{M^{n}}\left\{T\left(p y_{n}\right)-\rho f\left(y_{n}\right)+\rho N\left(y_{n}, y_{n}\right)\right\}\right]+\gamma_{n} s_{n}
\end{aligned}
$$

where $\left\{\alpha_{n}\right\},\left\{\beta_{n}\right\},\left\{\gamma_{n}\right\},\left\{e_{n}\right\},\left\{r_{n}\right\},\left\{s_{n}\right\}$ and $\left\{l_{n}\right\}$ are same in Algorithm 3.2, then

1) The sequence $\left\{u_{n}\right\}$ defined by Algorithm 3.2, converges strongly to unique solution $u^{*}$ of the problem (3),

2) If $\varepsilon_{n}=\alpha_{n} \Delta_{n}+\chi_{n}$ with $\sum_{n=0}^{\infty} \chi_{n}<+\infty$ and

$\lim _{n \rightarrow \infty} \Delta_{n}=0$, then $\lim _{n \rightarrow \infty} y_{n}=u^{*}$,

3) $\lim _{n \rightarrow \infty} y_{n}=u^{*}$ implies that $\lim _{n \rightarrow \infty} \varepsilon_{n}=0$.

\section{Conclusions}

The objective of this paper is to establish existence and uniqueness results of generalized nonlinear implicit quasi variational inclusion problem in Banach spaces. We developed the $T$-resolvent operator with $T$-accretive mapping by using the concepts of Fang and Huang [11] and Peng [10] and proved that the problem (1) is equivalent to a fixed point problem. On the basis of fixed point formulation we suggested perturbed iterative algorithm with errors and by the theory of Hick and Harder [20] we proved the convergence and stability of iterative sequences generated by algorithms.

A further attention is required for the study of variational inclusions that might provide useful mathematical tools to deal with the problems arising in mathematical sciences.

\section{REFERENCES}

[1] S. Adly, "Perturbed Algorithms and Sensitivity Analysis for a General Class of Variational Inclusions," Journal of Mathematical Analysis and Applications, Vol. 201, No. 2, 1996, pp. 609-630. doi:10.1006/jmaa.1996.0277

[2] Ya. Alber, "Metric and Generalized Projection Operators in Banach Spaces: Properties and Applications," In: A. Kartsatos, Ed., Theory and Applications of Nonlinear Operators of Monotone and Accretive Type, Marcel Dekker, New York, 1996, pp. 15-50.

[3] H. Attouch, "Variational Convergence for Functions and Operators," Applicable Mathematics Series, Pitman, Massachusetts, 1984.
[4] C. Baiocchi and A. Capelo, "Variational and QuasiVariational Inequalities Application to Free Boundary Problems," Wiley, New York, 1984.

[5] H. Y. Lan and R. U. Verma, "Iterative Algorithms for Nonlinear Fuzzy Variational Inclusions Systems with (A, $\eta)$-accretive Mappings in Banach Spaces," Advances in Nonlinear Variational Inequalities, Vol. 11, No. 1, 2006, pp. 15-30.

[6] R. U. Verma, "Generalized System for Relaxed Coercive Variational Inequalities and Projection Methods," Journal of Optimization Theory and Applications, Vol. 121, No. 1, 2004, pp. 203-210.

doi:10.1023/B:JOTA.0000026271.19947.05

[7] R. U. Verma, "Approximation Solvability of a New Class of Nonlinear Set-Valued Variational Inclusions Involving (A, $\eta$ )-Monotone Mappings," Journal of Mathematical Analysis and Applications, Vol. 337, No. 2, 2008, pp. 969975. doi:10.1016/j.jmaa.2007.01.114

[8] Z. B. Xu and G. F. Roach, "Characteristic Inequalities Uniformly Convex and Uniformly Smooth Banach Spaces," Journal of Mathematical Analysis and Applications, Vol. 157, No. 1, 1991, pp. 189-210. doi:10.1016/0022-247X(91)90144-O

[9] Z. B. Xu, "Inequalities in Banach Spaces with Applications," Nonlinear Analysis, Vol. 16, No. 12, 1991, pp. 1127-1138. doi:10.1016/0362-546X(91)90200-K

[10] W. Peng, "Set Valued Variational Inclusions with TAccretive Operators in Banach Spaces," Applied Mathematics Letters, Vol. 19, No. 3, 2004, pp. 273-282. doi:10.1016/j.aml.2005.04.009

[11] Y. P. Fang and N. J. Huang, "H-accretive Operators and Resolvent Operator Technique for Solving Variational Inclusions in Banach Spaces," Applied Mathematics Letters, Vol. 17, No. 6, 2004, pp. 647-653. doi:10.1016/S0893-9659(04)90099-7

[12] X. P. Ding, "Generalized Implicit Quasivariational Inclusions with Fuzzy Set Valued Mappings," Computer Mathematics with Applications, Vol. 38, No. 1, 1999, pp. 71-79.

[13] Y. P. Fang, N. J. Huang, J. M. Kang and Y. J. Cho, "Generalized Nonlinear Implicit Quasivariational Inclusions," Journal Inequalities and Applications, Vol. 3, 2005, pp. 261-275.

[14] N. J. Huang, "Mann and Ishikawa Type Perturbed Algorithms for Generalized Nonlinear Implicit Quasivaria- 
tional Inclusions," Journal of Mathematical Analysis and Application, Vol. 210, No. 1, 1997, pp. 88-101.

[15] X. P. Ding, "Perturbed Proximal Point Algorithms for Generalized Quasivariational Inclusions," Journal of Mathematical Analysis and Applications, Vol. 210, No. 1, 1997, pp. 88-101. doi:10.1006/jmaa.1997.5370

[16] S. H. Shim, S. M. Kang, N. J. Huang and Y. J. Cho, "Perturbed Iterative Algorithms with Errors for Completely Generalized Strongly Nonlinear Implicit Quasivariational Inclusions," Journal of Inequalities and Applications, Vol. 5, No. 4, 2000, pp. 381-395.

[17] S. S. Chang, "On Chidume's Open Questions and Approximate Solution of Multivalued Strong Mapping Equations in Banach Spaces," Journal of Mathematical Analysis and Applications, Vol. 216, No. 1, 1997, pp. 94-111. doi:10.1006/jmaa.1997.5661

[18] N. J. Huang, M. R. Bai, Y. J. Cho and S. M. Kang, "Generalized Nonlinear Mixed Quasivariational Inequalities," Computer Mathematics with Applications, Vol. 40, No. 2-3, 2000, pp. 205-215. doi:10.1016/S0898-1221(00)00154-1

[19] M. O. Osilike, "Stable Iteration Procedures for Strong
Pseudo-Contractions and Nonlinear Operators of the Accretive Type," Journal of Mathematical Analysis and Applications, Vol. 204, No. 3, 1996, pp. 677-692. doi:10.1006/jmaa.1996.0461

[20] A. M. Harder and T. L. Hicks, "Stability Results for Fixed Point Iteration Procedures," Mathematics Journal, Vol. 33, 1988, pp. 693-706.

[21] N. J. Huang and Y. P. Fang, "A Stable Perturbed Proximal Point Algorithm for a New Class of Generalized Strongly Nonlinear Quasivariational Like Inclusions," in Press.

[22] W. R. Mann, "Mean Value Methods in Iteration," Proceeding of American Mathematical Society, Vol. 4, 1953, pp. 506-510.

[23] Ishikawa, "Fixed Points and Iteration of a Non-Expansive Mapping in Banach Spaces," Proceeding of American Mathematical Society, Vol. 59, No. 1, 1976, pp. 65-71. doi:10.1090/S0002-9939-1976-0412909-X

[24] N. J. Huang, "Mann and Ishikawa Type Perturbed Iterative Algorithms for Generalized Nonlinear Implicit Quasivariational Inclusions," Computer Mathematics with Applications, Vol. 35, No. 10, 1998, pp. 1-7. 